\title{
Midkine as a novel biomarker for contrast-induced acute kidney injury
}

\author{
Mahmoud Abdelnabi MD, Abdallah Almaghraby MD, \\ Juthipong Benjanuwattra MD, Mohamed Ahmed MD
}

\begin{abstract}
Contrast-induced acute kidney injury (Cl-AKI) is an intrinsic kidney injury caused by contrast injection in susceptible individuals. Although the pathophysiological mechanisms by which contrast agents induce kidney injury have not been completely explained, direct and indirect effects, as well as hemodynamic perturbations, have been suggested. Renal effects develop immediately after contrast injection, and, theoretically, they could be detected early by using a sensitive biomarker. Recently, studies have been conducted to determine specific biomarkers to guide the early diagnosis of $\mathrm{Cl}-\mathrm{AKI}$ and thus improve the outcomes of these patients. Midkine (MK) is a heparin-binding growth factor that balances cell growth, survival, and migration. It has an antiapoptotic activity in nephrogenesis. Midkine has been investigated as an early biomarker for Cl-AKI in patients undergoing percutaneous coronary intervention (PCI) for stable angina and was significantly higher in Cl-AKI patients post PCU. However, more studies are needed to validate its efficacy in the early detection of $\mathrm{Cl}-\mathrm{AKI}$.
\end{abstract}

Keywords: Contrast-induced nephropathy, contrast-induced acute kidney injury, midkine, biomarker.

\section{BACKGROUND}

Contrast-induced acute kidney injury (Cl-AKI) accounts for one-third of all hospital-acquired acute kidney injuries $(\mathrm{AKI})$ with an incidence of $1-2 \%$ in the general hospitalized population and up to $50 \%$ in highrisk individuals. ${ }^{1}$ Contrast-induced acute kidney injury is defined as AKI that develops within 24-48 hours after exposure to contrast media and exclusion of other causes. $^{2}$ Contrast-induced acute kidney injury can be diagnosed if there is a $25 \%$ relative increase or a $0.5 \mathrm{mg} / \mathrm{dl}$ absolute rise in serum creatinine (SCr) level within 72 hours after contrast exposure with no other alternative explanation. ${ }^{1}$ Certain limitations in the diagnostic criteria exist, including the absence of functional

Corresponding author: Mahmoud Abdelnabi Contact Information: Mahmoud.Abdelnabi@ttuhsc.edu DOI: 10.12746/swrccc.v10i42.1001 assessment, such as changes in urine output, which is used in the Risk, Injury, Failure, Loss of kidney function, and End-stage kidney disease (RIFLE), ${ }^{3}$ Acute Kidney Injury Network (AKIN), ${ }^{4}$ and Kidney Disease Improving Global Outcomes (KDIGO) ${ }^{5}$ classifications. A slight increase in $\mathrm{SCr}$, less than $0.5 \mathrm{mg} / \mathrm{dl}$, was also found to correlate with adverse events. ${ }^{2}$ Nevertheless, this definition is widely used in most Cl-AKI studies, and it correlates well with adverse clinical endpoints. ${ }^{6}$

Given the progressive reduction in hospital length of stay for diagnostic and interventional procedures, detection of $\mathrm{Cl}-\mathrm{AKI}$ in this setting will continue to be challenging. In addition, emerging evidence suggests that SCr lacks the sensitivity to diagnose occult but clinically relevant $\mathrm{AKI},{ }^{7}$ and increased $\mathrm{SCr}$ levels provide no information regarding the etiology or anatomical location of renal injury. ${ }^{8}$ Since the clinical manifestations and treatment of $\mathrm{Cl}-\mathrm{AKI}$ are, in fact, the same as $\mathrm{AKI}$ induced by other causes, the biomarkers studied to make a rapid diagnosis of AKI-induced by other causes 
could also be used to predict $\mathrm{Cl}-\mathrm{AKI} .{ }^{9}$ For these reasons, studies to identify and validate novel biomarkers with higher sensitivity for early $\mathrm{Cl}-\mathrm{AKI}$ are important.

Midkine (MK) is a heparin-binding growth factor that has a role in organogenesis and several pathologic conditions; it belongs to the pleiotrophin/MK family. ${ }^{10}$ Human MK is a $13 \mathrm{kDa}$ protein with 121 amino acids. ${ }^{10}$ Despite abundant expression of MK during embryogenesis, evidence suggests that, during adulthood, MK is expressed only in certain organs, including the kidneys, intestines, and bronchial epithelium. ${ }^{10}$ Midkine is involved in many physiological processes, such as growth and differentiation, reproduction, and tissue repair. ${ }^{11}$ Midkine is also involved in inflammation, as is evident in in-vivo models of arterial restenosis, rheumatoid arthritis, ischemic renal injury, cisplatin-induced tubulointerstitial nephropathy, and diabetic nephropathy. ${ }^{12}$ The expression of MK is reportedly upregulated following ischemic injury in various organs. ${ }^{10}$ In the healthy kidney, MK is expressed in the glomeruli, interstitium, proximal and distal tubular cells, and endothelial cells. ${ }^{11}$ However, the expression of MK is markedly upregulated in the epithelium of the proximal tubules, through the activation of hypoxia-inducible factor- $1 \alpha$ (HIF-1 $\alpha$ ) during oxidative stress. ${ }^{13}$ In this mini-review, we have summarized the evidence suggesting a role for MK in renal disease and suggest that MK could be an early indicator for diagnosing $\mathrm{Cl}-\mathrm{AKI}$.

\section{MIDKINE EXPRESSION IN NEPHROGENESIS}

During the mid-gestational period of embryogenesis, MK is abundantly expressed in metanephrogenic blastema due to modulation by retinoic acids. The growth of the embryonic kidney depends on mesenchymal-epithelial interactions. It has been proposed that MK is implicated in the development of renal epithelial tissues, since in-vitro inhibition of MK led to a decrease in the formation of nephrons by approximately $50 \% .^{14}$

\section{MIDKINE IN RENAL DISEASE}

The pathophysiological effects of MK in renal disease are diverse, varying from the development of acute kidney injury (AKI) to chronic kidney disease (CKD) progression, and it is also involved in renal ischemia and diabetic nephropathy. ${ }^{15}$ Several mechanisms have been proposed as the underlying pathophysiology of MK-associated chronic kidney disease, including inflammatory cell infiltration through chemokines and progressive fibrosis due to the activation of transforming growth factor beta- $1 .{ }^{16}$ From a study of 197 patients with pre-dialysis CKD, the levels of serum and urine MK significantly increased in a step-wise manner across CKD stage III-V ${ }^{16}$ which may be explained by reduced renal excretion, increased renal production from damaged tubules, or chronic inflammatory state from CKD itself. ${ }^{16}$ In this study, the median serum level of MK in normal volunteers was $754 \mathrm{pg} / \mathrm{ml}$. There was a positive correlation between the level of MK and the severity of CKD. The reported mean serum level of MK was as high as $4816 \mathrm{pg} / \mathrm{ml}$ in CKD-V patients. ${ }^{16}$ Limited evidence exists regarding the implication of MK in dialysis patients, but it was shown that the administration of heparin, but not non-heparin anticoagulants, in those patients resulted in a rapid elevation of the serum MK levels. ${ }^{16}$

Diabetic nephropathy is one of the most frequent causes of morbidity and mortality in diabetic patients. Long-standing hyperglycemia induces severe endothelial dysfunction, oxidative stress, and inflammation, leading to renal dysfunction. ${ }^{14}$ It has been shown that the levels of MK are markedly elevated in children with type 1 diabetes mellitus, particularly in those with albuminuria. ${ }^{17}$

Renal ischemia is associated with systemic involvement and can lead to multiorgan failure..$^{18}$ During renal ischemia, energy depletion in renal epithelial cells results in cytoskeletal disruption, aberrant cell polarity, and cellular death. ${ }^{18}$ In addition, indirect injury can be explained by the MK-mediated upregulation of macrophage inflammatory protein-2 (MIP-2) and subsequent recruitment of inflammatory cells. ${ }^{18} \mathrm{~A}$ previous study showed that extreme ATP and GTP consumption is associated with necrotic and apoptotic cell death, respectively. ${ }^{19}$ Endothelial cell malfunction causes vascular congestion and edema, blood flow impairment, and the migration of inflammatory cells, such as neutrophils and macrophages to the injured kidney followed 
by a release of cytokines, reactive oxygen species, proteases, myeloperoxidase, and other chemokines to cause additional damage. ${ }^{18}$ Following renal ischemic/ reperfusion injury, it has been shown that the synthesis of MK is significantly increased in diseased tubules, which is associated with elevated levels of MIP-2. ${ }^{20}$ Inhibiting both MK and MIP2 results in decreased infiltration of neutrophils and macrophages, thus reducing the intensity of tubulointerstitial injury. ${ }^{18,20}$

Using $\mathrm{MK}$ as a renal biomarker in $\mathrm{Cl}-\mathrm{AKI}$ has been studied in patients with stable angina undergoing percutaneous coronary intervention $(\mathrm{PCl})$. It was demonstrated that the serum levels of MK were significantly higher in $\mathrm{Cl}-\mathrm{AKI}$ patients. ${ }^{21} \mathrm{An}$ increase in serum MK was detected as early as 2 hours following $\mathrm{PCl}$, with the mean serum levels of $2,460 \mathrm{pg} / \mathrm{ml}$, and it remained significantly elevated for 8 hours after the procedure. ${ }^{21}$ Ahmed et al. ${ }^{22}$ studied the use of serum MK as an early predictor of CI-AKI in 100 patients with eGFR $<60 \mathrm{ml} / \mathrm{min} / 1.73 \mathrm{~m}^{2}$ undergoing $\mathrm{PCl}$ for the acute coronary syndrome (ACS). Serum MK levels were measured before $\mathrm{PCl}$ and 2 and 24 hours after $\mathrm{PCl}$. The results showed that the serum levels of $\mathrm{MK}$ at 2 hours post-PCl can be used as an early predictor of $\mathrm{Cl}-\mathrm{AKI}$ in ACS patients undergoing $\mathrm{PCl}$. Jones et al. stated that MK might be more sensitive in AKI diagnoses than other biomarkers. Comparing MK measurement with other biomarkers can increase the sensitivity for AKI diagnosis. ${ }^{23}$ Nevertheless, more studies are necessary to validate using MK in $\mathrm{Cl}-\mathrm{AKI}$.

\section{Conclusion}

Overall, the pathogenesis of Cl-AKI is not yet completely understood. Although often a brief injury, $\mathrm{Cl}-\mathrm{AKI}$ may progress to significant persistent renal impairment, end-stage kidney disease, and adverse cardiovascular sequelae. Current studies are largely focused on $\mathrm{Cl}-\mathrm{AKI}$ prevention in susceptible patients. However, no consensus regarding the treatment of $\mathrm{Cl}-\mathrm{AKI}$ has been developed once it occurs. In current clinical practice, $\mathrm{SCr}$ and assessment of urine output are the most commonly used measures of renal dysfunction, but they have limited sensitivity and specificity. Serum creatinine is a marker of function, not injury, and it may take days after injury to increase, thereby limiting its usefulness in the early detection of $\mathrm{Cl}-\mathrm{AKI}$. It is possible that the renal injury starts immediately after contrast administration. Sensitive early biomarkers may be able to detect kidney injury promptly, and to this effect, much effort has been made in recent years to identify the appropriate marker. The development of novel biomarkers, such as MK, is promising and may provide more rapid detection of $\mathrm{Cl}-\mathrm{AKI}$ before the expected rise in SCr and give a diagnosis in hours, rather than days, which could lead to improved patient outcomes.

Article citation: Abdelnabi M, Almaghraby A, Benjanuwattra J, Ahmed M. Midkine as a novel biomarker for contrast-induced acute kidney injury. The Southwest Respiratory and Critical Care Chronicles 2022;10(42):12-15 From: Department of Internal Medicine (MA, JB), Texas Tech University Health Sciences Center, Lubbock, Texas; Cardiology and Angiology Unit (MA), Clinical and Experimental Internal Medicine Department, Medical Research Institute, Alexandria University, Alexandria, Egypt; Cardiology Department (AA), Faculty of Medicine, Alexandria University, Alexandria, Egypt; Critical Care Department (MA), Manchester Royal Infirmary, Manchester University NHS Foundation Trust, Manchester, UK

Submitted: 1/10/2022

Accepted: $1 / 14 / 2022$

Conflicts of interest: none

This work is licensed under a Creative Commons Attribution-ShareAlike 4.0 International License.

\section{REFERENCES}

1. Mehran R, Nikolsky E. Contrast-induced nephropathy: definition, epidemiology, and patients at risk. Kidney Inter Suppl 2006;69:S11-S15.

2. Khwaja A. KDIGO clinical practice guidelines for acute kidney injury. Nephron Clin Prac 2012;120:c179-c184.

3. Bellomo R, Ronco C, Kellum JA, et al. Acute renal failuredefinition, outcome measures, animal models, fluid therapy and information technology needs: the Second International 
Consensus Conference of the Acute Dialysis Quality Initiative (ADQI) Group. Crit Care 2004;8(4):1-9.

4. Mehta RL, Kellum JA, Shah SV, et al. Acute Kidney Injury Network: report of an initiative to improve outcomes in acute kidney injury. Crit Care 2007;11(2):1-8.

5. Kellum JA, Lameire N. Diagnosis, evaluation, and management of acute kidney injury: a KDIGO summary (Part 1). Crit Care 2013;17(1):1-15.

6. Rear R, Bell RM, Hausenloy DJ. Contrast-induced nephropathy following angiography and cardiac interventions. Heart 2016;102(8):638-648.

7. Ling W, Zhaohui N, Ben H, et al. Urinary IL-18 and NGAL as early predictive biomarkers in contrast-induced nephropathy after coronary angiography. Nephron Clin Pract 2008;108(3): c176-c181.

8. Soni SS, Ronco C, Katz N, et al. Early diagnosis of acute kidney injury: the promise of novel biomarkers. Blood purif 2009;28(3):165-174.

9. Andreucci M, Faga T, Riccio E, et al. The potential use of biomarkers in predicting contrast-induced acute kidney injury. Int J Nephrol Renovasc Dis 2016;9:205.

10. Muramatsu T. Structure and function of midkine as the basis of its pharmacological effects. Br J Pharmacol 2014;171(4): 814-826.

11. Bădilă E, Daraban AM, Țintea E, et al. Midkine proteins in cardio-vascular disease.: Where do we come from and where are we heading to? Europ J Pharmacol 2015;762:464-471.

12. Kosugi T, Yuzawa Y, Sato W, et al. Midkine is involved in tubulointerstitial inflammation associated with diabetic nephropathy. Lab Invest 2007;87(9):903-913.

13. Şalaru DL, Arsenescu-Georgescu C, Chatzikyrkou C, et al. Midkine, a heparin-binding growth factor, and its roles in atherogenesis and inflammatory kidney diseases. Nephrol Dial Transplant 2016;31(11):1781-1787.
14. Sato W, Sato Y. Midkine in nephrogenesis, hypertension and kidney diseases. Br J Pharmacol 2014;171(4):879-887.

15. Kato K, Kosugi T, Sato W, et al. Growth factor Midkine is involved in the pathogenesis of renal injury induced by protein overload containing endotoxin. Clin Exp Nephrol 2011;15(3): 346-354.

16. Campbell VK, Anstey CM, Gately RP, et al. Urine and serum midkine levels in an Australian chronic kidney disease clinic population: an observational study. BMJ open 2017;7(9): e014615. DOI: 10.1136/bmjopen-2016-014615

17. Metwalley KA, Farghaly HS, Gabri MF, et al. Midkine: utility as a predictor of early diabetic nephropathy in children with type 1 diabetes mellitus. J Clin Res Pediatr Endocrinol 2021;13(3):293-299.

18. Kosugi T, Sato W. Midkine and the kidney: health and diseases. Nephrol Dial Transplant 2012;27(1):16-21.

19. Padanilam BJ. Cell death induced by acute renal injury: a perspective on the contributions of apoptosis and necrosis. Am J Physiol Renal Physiol 2003;284(4):F608-F627.

20. Sato W, Takei Y, Yuzawa $Y$, et al. Midkine antisense oligodeoxyribonucleotide inhibits renal damage induced by ischemic reperfusion. Kidney Int 2005;67(4):1330-1339.

21. Malyszko J, Bachorzewska-Gajewska H, Koc-Zorawska E, et al. Midkine: a novel and early biomarker of contrastinduced acute kidney injury in patients undergoing percutaneous coronary interventions. BioMed Res Int 2015;2015 DOI: $10.1155 / 2015 / 879509$

22. Ahmed MA, Abdelnabi M, Almaghraby A. CRT-100.94 Midkine as an early predictor of contrast-induced acute kidney injury. JACC Cardiovasc Interv 2020;13(4 Supplement S): S26-S26.

23. Jones D. Measuring midkine: the utility of midkine as a biomarker in cancer and other diseases. Br J Pharmacol 2014; 171(12):2925-2939. 